\title{
Seroprevalence of torch infections among complicated pregnancies in Atbara river Nile state - Sudan
}

\begin{abstract}
Objective: The aim of this study was to find the seroprevalence of TORCH infection among volunteered pregnant women and to find out the correlation between TORCH infection and age, complicated pregnancies and education level.

Methods: A descriptive cross-sectional laboratory-based study carried out between March to June 2012 at Atbara River Nile State northern Sudan. The statistical descriptive determined in means of percentages. Chi-square used for trend analysis and calculates p-values.

Results: A total of (75) voluntary pregnant women, Age between 15-55 years. The most common age group was 26-35 years (37/75) 49\%.Serological evidence of Toxoplasmosis were positive for (22/75) $29 \%$. All volunteered pregnant women were negative for other components of TORCH complex. Study showed non-significant correlation between age, education level and complicated pregnancies, P-value $0.13,0.43$ and 0.51 respectively.

Conclusion: Low prevalence of Toxoplasmosis and absent of serological evidence of other TORCH complex among volunteered pregnant women in Atbara has been documented. Insignificant correlation between toxoplasmosis and age, education level as well as number of complicated pregnancies reported. This attributed, in part, to the small sample size and using screening tool as diagnostic modalities.
\end{abstract}

Keywords: Torch, toxoplasmosis, atbara, sudan
Volume I0 Issue I - 202I

\author{
Mohammed Ahmed Ibrahim Ahmed,' Hanaa \\ Elzain Musaad, ${ }^{2}$ Nahla Ahmed Mohammed \\ Abdurrahman, ${ }^{3}$ Wadei Mohammed Yasin, ${ }^{4}$ \\ Mosab Nouraldein Mohammed Hamad, ${ }^{5}$ \\ Nassreldeen Khalid Abdelrahman, ${ }^{6}$ Emad \\ Ibrahim Osman ${ }^{7}$ \\ 'Faculty of Medicine, Department Microbiology, Nile Valley \\ University, Sudan \\ ${ }^{2}$ Laboratory Technologist, Ministry of Health, River Nile State, \\ Sudan \\ ${ }^{3}$ Faculty of Medicine, Department Biochemistry Atbara, Nile \\ Valley University, Sudan \\ ${ }^{4}$ Faculty of Medicine, Department of pathology Science, Sudan \\ International University, Sudan \\ ${ }^{5}$ Department of Medical Laboratory Sciences, Faculty of Health \\ Science, Elsheikh Abdallah Elbadri University, Sudan \\ ${ }^{6}$ University of Al Fashir, Faculty of Medical Laboratory Science, \\ Sudan \\ ${ }^{7}$ Faculty of Medical Laboratory Science, Elrazi University, Sudan
}

\begin{abstract}
Correspondence: Mosab Nouraldein Mohammed Hamad, Phylum of Medical Parasitology, Department of Medical Laboratory Sciences, Faculty of Health Science, Elsheikh Abdallah Elbadri University, Berber, Sudan.

Tel 00249929194I37, Email musab.noor13@gmail.com
\end{abstract}

Received: Januray II, 2021 | Published: February 12, 202 I
Abbreviations: CMV: cytomegalovirus; HSV: herpes simplex virus; $\mathrm{BOH}$ : Bad obstetrics history; $\mathrm{CS}$ : caesarian section; ELISA: enzyme linked immunosorbent assay

\section{Introduction}

The TORCH infections are a collection of viral, bacterial, and protozoan infections that can access to the fetal blood stream transplacentally via the chorionic villi and cause serious fetal anomalies or abortion. Vertical transmission may happen when throughout pregnancy or infrequently at the time of delivery via maternal-to-fetal transfusion. ${ }^{1}$ Major infections caused by TORCHToxoplasma gondii, Rubella virus, cytomegalovirus (CMV), and herpes simplex virus (HSV), are the main reasons of $\mathrm{BOH} .{ }^{2}$ Infections often occur before the woman realizes that she is pregnant or seeks medical care. The primary infection is probable to have a more significant influence on fetus than recurring infection and may lead to congenital anomalies, spontaneous abortion, intrauterine fetal death, intrauterine growth retardation, prematurity, stillbirth, and live born infants with the proof of disease. ${ }^{3}$ Most of the TORCH infections cause mild maternal morbidity but have grave fetal consequences. ${ }^{4}$ Capability of the fetus to fight infectious organisms is incomplete and the fetal immune system is unable to stop the spreading of infectious organisms to several tissues. ${ }^{5}$
TORCH infections in the mother are communicable to fetus in the womb or throughout the birth progression and lead to a cluster of symptomatic birth disorders. Several sensitive and specific tests are accessible for serological diagnosis of TORCH complex; ${ }^{6}$ though, ELISA test is more regularly used for its sensitivity. An effort made to find out the correlation of TORCH infection throughout pregnancy in the Iraqi population. ${ }^{7}$ Toxoplasma gondii is an obligate intracellular protozoan parasite, which connected to one of the most predominant chronic infections affecting one-third of the world's population. ${ }^{7}$ Commonness of toxoplasmosis between aborted women in Atbara in Atbara was $33.6 \%$ investigated by Enzyme Linked Immune Sorbent Assay (ELISA). ${ }^{8}$ Eruption of toxoplasmosis accompanying with low education level as well as other risk factors like water pollution, raw milk and undercooked meat. ${ }^{9}$

\section{Patients and methods}

\section{Study Design}

A descriptive cross-sectional laboratory-based study conducted in the period from March- June 2012.

\section{Study Area}

The study conducted in River Nile State, Atbara. 


\section{Study population}

All women of history of complicated pregnancy, who volunteered to take part, included.

\section{Study variable}

The dependent variable of this study was TORCH rapid test result, because the independent variables were age, education level, number of normal and complicated pregnancies.

\section{Sampling technique, and sample size}

Five $\mathrm{ml}$ of blood drawn from each subject, sera separated, and tested for TORCH using rapid Dip-strip (manufactured in the UK by fortress diagnostic LTD). Blood collected from 75 volunteered pregnant women in free anticoagulant plain containers a data collected by a structural interview questionnaire. The aims of the study confirmed to all volunteers and their permission obtained.

\section{Ethics declarations}

Ethical permission obtained from ministry of health. Participation was optional; there would be no penalty for refusal. A full explanation of the goals and methods of the study elucidated. Informed written consents obtained from all participants before being enrolled.

\section{Data Analysis}

The data generated coded, validated, and analyzed using Statistical Package for Social Sciences (SPSS) version 20 (IBM Statistics, SPSS Inc., Chicago, IL).Pearson chi-squared test was used to test for significance between proportions. P-Values below 0.05 were considered statistically significant. The main variables analyzed were: age, education level, and number of normal and complicated pregnancies.

\section{Results}

A total of 75 volunteered pregnant women from Atbara River Nile State, enrolled in this study, the ages of participants ranged from 15 to 55 years. Twenty two of seventy-five (22/75) $29 \%$ were positive for toxoplasmosis (Table 1). Study showed statistically insignificant correlation between toxoplasmosis and age, education level and number of complicated pregnancies $(\mathrm{P}=0.13)(\mathrm{P}=0.43)(\mathrm{P}=0.51)$. But majority of positive serological evidence of toxoplasmosis among age of (26-35) was (14/37) $37 \%$ (Table 2).

Table I Distribution of TORCH test

\begin{tabular}{llll}
\hline Test & Positive & Negative & Percent \\
\hline Toxoplasmosis & $22(29 \%)$ & $53(61 \%)$ & $100 \%$ \\
Rubella Virus & $0(0.0 \%)$ & $75(100.0 \%)$ & $100 \%$ \\
Cytomegalo Virus & $0(0.0 \%)$ & $75(100.0 \%)$ & $100 \%$ \\
Herpes simplex 2 & $0(0.0 \%)$ & $75(100.0 \%)$ & $100 \%$ \\
\hline
\end{tabular}

\section{Discussion}

Findings of this study show the low prevalence of toxoplasmosis among volunteered pregnant women, twenty two of seventy-five (22/75) $29 \%$ were positive for toxoplasmosis, this was low comparable to that reported in Atbara which revealed (33.6\%) of study group were positive for toxoplasmosis by Latex Agglutination Test (LAT). ${ }^{8}$ This attributed the small sample size, different characteristics of study group, different type of laboratory diagnostic modalities, or in part attributed to good health facilities of prenatal care provided by health authorities. This finding may suggest the need to study prevalence of toxoplasmosis and other components of TORCH complex by ELISA as well as molecular techniques. This point necessitates studying large groups in Atbara for toxoplasmosis as well as TORCH complex because sample size of our study not allows for generalization of the results to the population. Significant risk factors for toxoplasmosis like low education level was not recognized in this study. Association of toxoplasmosis and low education level is well documented in the literature. ${ }^{8}$ Despite the well-documented association between low education level and high sero-detection of toxoplasmosis in literature, low education level was not found with toxoplasmosis in this particular study. This attributed, in part, to the small group of study participants. Furthermore, there are some studies indicating that educated people are less likely to have serological evidence of toxoplasmosis. ${ }^{9-11}$

Table 2 Correlation between TORCH and independent variables

\begin{tabular}{lllll}
\hline Characteristic & Variable & Number & Percent \% & P-value \\
\hline \multirow{4}{*}{ Age group } & $15-25$ & $13 / 75$ & $17.30 \%$ & \\
& $26-35$ & $37 / 75$ & $49.30 \%$ & \\
& $36-45$ & $20 / 75$ & $26.70 \%$ & 0.13 \\
& $46-55$ & $5 / 75$ & $6.70 \%$ & \\
& Illiterate & $7 / 75$ & $9.30 \%$ & \\
Education level & Basic & $31 / 75$ & 41.3 & 0.43 \\
& High school & $24 / 75$ & $32.00 \%$ & \\
& University & $13 / 75$ & $17.30 \%$ & \\
Complicated & Abortion & $52 / 75$ & $69.30 \%$ & \\
pregnancy & Dead CS & $1 / 55$ & $98.20 \%$ & 0.51 \\
& Congenital CS & $1 / 55$ & $98.20 \%$ & \\
\hline
\end{tabular}

High prevalence of toxoplasmosis among study group (2635 ) without significant correlation reported in this particular study. In other study implemented in Southwest Ethiopia age was much associated with gondii seropositivity ${ }^{11}$ this finding may attributed to small selected group. History of abortion as index of complicated pregnancy reported in 52 of study group, without significant association with toxoplasmosis $(\mathrm{P}=0.51)$, the association between complicated pregnancy and toxoplasmosis documented in study conducted in Australia. ${ }^{11}$ This particular study had some limitations. A positive test measured positive for the goal of this study. There was no confirmatory test performed. So, false positives cannot be omitted, and that the true infectious disease rates lower than the stated. ${ }^{12}$

\section{Conclusion}

Low occurrence of Toxoplasmosis and lack of serological proof of further TORCH complex between volunteered pregnant women in Atbara has been documented. Irrelevant correlation between toxoplasmosis and age, education level as well as some complex pregnancies stated. This credited, partly, to the small sample size and using screening tool as diagnostic modalities. Further studies required with large sample size and diverse diagnostic methods.

\section{Conflicts of interest}

The authors declare that there are no conflicts of interest about this study. 


\section{Author's contributions}

The authors have contributed equally to the study design and manuscript write-up. All authors read and approved the last version of the manuscript.

\section{Acknowledgments}

Our special appreciation goes to the study participants. The authors wish to acknowledge staff of Modern Specialized Laboratory for their technical support.

\section{Funding}

None

\section{References}

1. Kumar V, Abbas AK, Fausto N, et al. Robbins \& Cotran, Pathologic Basis of Disease (8th Edition). Philadelphia, PA: Elsevier. 2009: 480.

2. McCabe R, Remington JS. Toxoplasmosis, the time has come. New Eng $J$ Med. 1988;318(5):313-315.

3. Maruyama Y, Sameshima H, Kamitomo M, et al. Fetal manifestations and poor outcomes of congenital cytomegalovirus infections: possible candidates for intrauterine antiviral treatments. $J$ Obst Gynae. 2007;33(5):619-613.

4. Stegmann BJ, Carey JC. TORCH infections. Toxoplasmosis, other (syphilis, varicella-zoster, parvovirus B19), Rubella, cytomegalovirus (CMV), and herpes infections. Cur Women's Health Reports. 2002;2(4):253-258.
5. Mladina N, Mehikic G, Pasic A. Torch infections in mothers as a cause of neonatal morbidity. Med Arh. 2000;54(5-6):273-276.

6. Stern H, Tacker SM. A prospective study of cytomegalovirus infection in pregnancy. Br Med J. 1973;2(5861):268-270.

7. Jones JL, Kruszon-Moran D, Wilson M, et al. Toxoplasma gondii infection in the United States: seroprevalence and risk factors. $\mathrm{Am} \mathrm{J}$ Epidemiol. 2001;154(4):357-365.

8. Taha RKM, Hamad MNM, Taha KM, et al. Seroprevalence of toxoplasmosis between aborted ladies in Atbara district, Sudan. MOJ Womens Health.2019;8(1):86-87.

9. Fernanda PF, Eloiza TC, Aline KSP, et al. Patterns of Transmission and Sources of Infection in Outbreaks of Human Toxoplasmosis. Emerg Infect Dis. 2019;25(12):2177-2182.

10. Mustafa M, Fatima F, Abubaker M, et al. Prevalence and risk factors profile of seropositive Toxoplasmosis gondii infection among apparently immunocompetent Sudanese women. BMC Res Notes. 2019;12(1):279.

11. Zeleke AJ, Melsew YA. Seroprevalence of Toxoplasma gondii and associated risk factors among HIV-infected women within reproductive age group at Mizan Aman General Hospital, Southwest Ethiopia :a cross sectional study. BMC Res Notes. 2019;10(1):70.

12. Andrea RP, David CK, Larry S, et al. Congenital toxoplasmosis in Austria: Prenatal screening for prevention is cost-saving. PLoS Negl Trop Dis.2017;11(7): e0005648. 